\title{
Resonant scattering probes in the terahertz range
}

\author{
Thomas Siday, Michele Natrella, Jiang Wu, Huiyun Liu and Oleg Mitrofanov \\ Electronic and Electrical Engineering, University College London, London, WC1E 7JE
}

\begin{abstract}
We propose and demonstrate a novel near-field probe, resonant at terahertz ( $\mathrm{THz})$ frequencies. The probe is a conical metallic tip, with length similar to the $\mathbf{T H z}$ wavelength, fabricated directly to the surface of a radially-polarized $\mathrm{THz}$ source. High field confinement is seen at the apex of the probe. Frequency dependent scattering efficiency is observed, likely related to dipolar resonances along the length of the probe.
\end{abstract}

\section{INTRODUCTION}

$\mathrm{T}$ $\mathrm{Hz}$ near-field imaging has progressed significantly in recent years, showcasing spatial resolution better than $100 \mathrm{~nm}$ [1]. However, the sensitivity of the technique is limited by low efficiency in converting the near-field interaction at the apex of the probe to the far field. In the infra-red (IR) range, scattering probes with length $L$ similar to their operating wavelength $\lambda$ are seen to enhance scattering efficiency by acting as a resonant antenna [2]. In most cases, $\mathrm{THz}$ scattering probe microscopy utilizes a needle $(L>>\lambda)$ or atomic force microscope (AFM) cantilever $(L<<\lambda)$ as a near-field probe. To apply the principle of resonant scattering probes to the $\mathrm{THz}$ range we demonstrate a novel $\mathrm{THz}$ scattering probe, fabricated from Indium, with variable length between $\sim 50 \mu \mathrm{m}$ and $1000 \mu \mathrm{m}$, and apex diameter $\sim 50-150 \mathrm{~nm}$. The ideal half wave dipole model predicts the tips resonate between 0.15 and $3 \mathrm{THz}$.

The indium tips are fabricated on a wafer containing InAs, and excited by radially polarized $\mathrm{THz}$ pulses [3], which are guided along the length of the probe. A schematic of the probe in a THz scattering probe microscopy configuration is shown in fig. 1), with a scanning electron micrograph (SEM) of an exemplary probe inset.

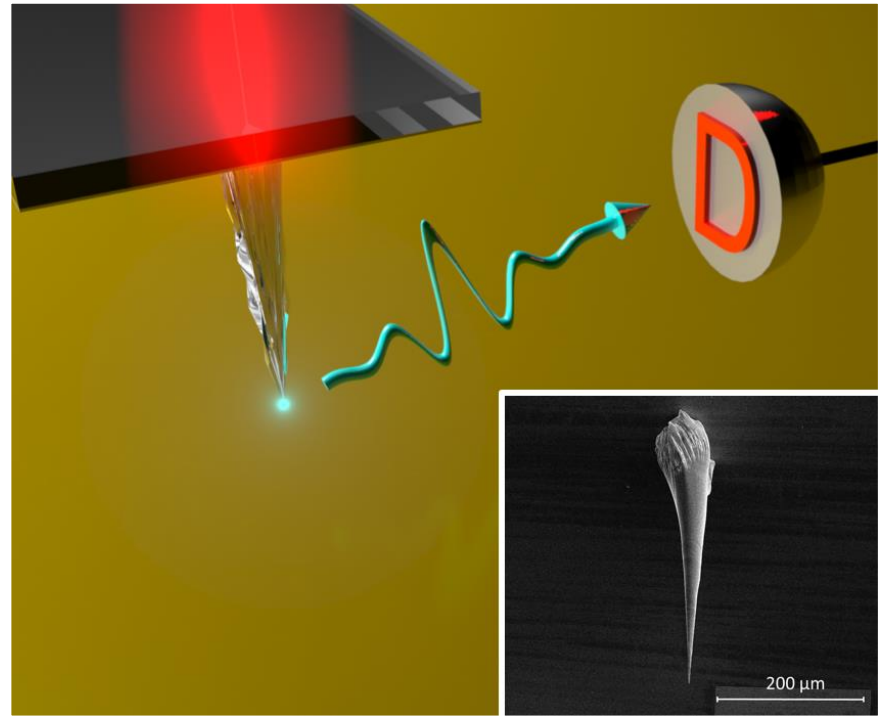

Fig. 1. Visualization of the indium tip configured for scattering probe microscopy. Detector D is placed in the far field. Inset: scanning electron micrograph of a $270 \mu \mathrm{m}$ long Indium tip with a $50 \mathrm{~nm}$ apex diameter.

\section{RESULTS}

The spatial field confinement at the apex of the indium tip is evaluated experimentally using aperture-type near-field microscopy integrated into a $\mathrm{THz}$ time-domain spectroscopy system (THz TDS). The field at the apex is seen to be confined to at least $4 \mu \mathrm{m}$, the resolution of the aperture probe [4]. Finite-difference time-domain simulations predict that the true field confinement is in fact comparable to the tip apex diameter.

Using the THz TDS system, with detection in the far field, it is possible to identify the field scattered only from the Indium tip (fig. 2). Compared to the emission spectrum from the $\mathrm{THz}$ source, the spectrum shows two peaks. These are likely to be resonances related to the shaft of the tip. We suggest that the lower frequency peak is related to the half-wave dipole resonance, which can be efficiently excited with a radial source [5].

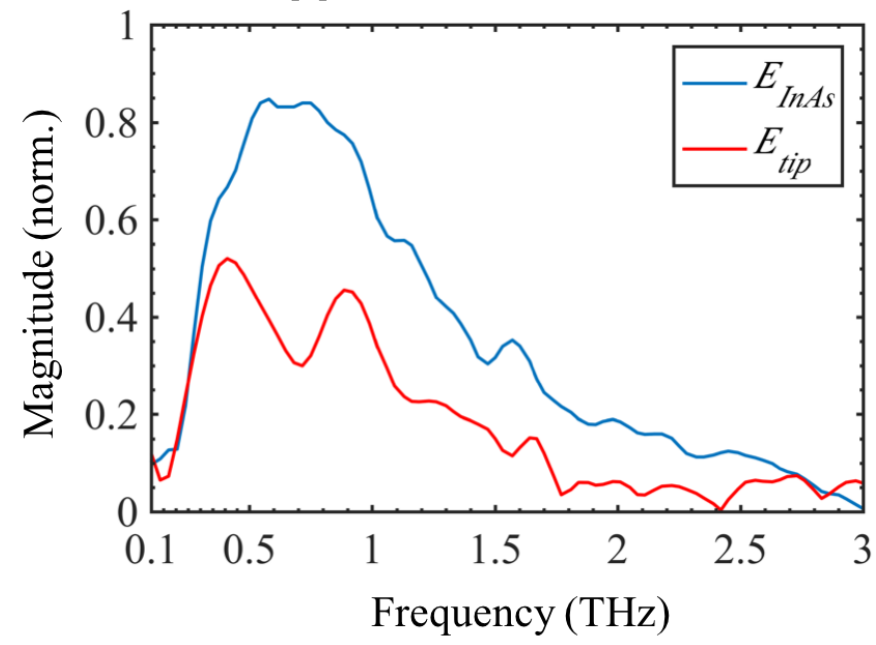

Fig. 2. Spectrum measured by a detector placed $\sim 5 \mathrm{~mm}$ from the tip, showing emission from InAs only (blue line) and the emission from the tip only (red line).

\section{REFERENCES}

[1] A. J. Huber, F. Keilmann, J. Wittborn, J. Aizpurua, and R. Hillenbrand, 'Terahertz Near-Field Nanoscopy of Mobile Carriers in Single Semiconductor Nanodevices', Nano Lett., vol. 8, no. 11, pp. 3766-3770, Nov. 2008.

[2] F. Huth et al., 'Resonant Antenna Probes for Tip-Enhanced Infrared Near-Field Microscopy', Nano Lett., vol. 13, no. 3, pp. 1065-1072, Mar. 2013.

[3] R. Mueckstein et al., 'Near-Field Analysis of Terahertz Pulse Generation From Photo-Excited Charge Density Gradients', IEEE Trans. Terahertz Sci. Technol., vol. 5, no. 2, pp. 260-267, 2015.

[4] O. Mitrofanov et al., 'Near-Field Spectroscopy and Imaging of Subwavelength Plasmonic Terahertz Resonators', IEEE Trans. Terahertz Sci. Technol., vol. 6, no. 3, pp. 382-388, 2016.

[5] S. Tuccio et al., 'Direct determination of the resonance properties of metallic conical nanoantennas', Opt. Lett., vol. 39, no. 3, pp. 571-573, Feb. 2014. 